\title{
Evolution of Matrix-Twin Interfaces of (1012) Twin in Magnesium
}

\author{
A. Ostapovets ${ }^{a, *}$ AND A. Serra ${ }^{b}$ \\ ${ }^{a}$ Central European Institute of Technology - Institute of Physics of Materials (CEITEC-IPM), \\ Academy of Sciences of the Czech Republic, Žižkova 22, 61662 Brno, Czech Republic \\ ${ }^{b}$ Department of Civil \& Environmental Engineering, Universitat Politecnica de Catalunya, \\ Jordi Girona 1-3, 08034 Barcelona, Spain
}

\begin{abstract}
Recently, the presence of basal-prismatic interfaces in hexagonal close packed metals became subject of intensive investigation. We model the $\{10 \overline{1} 2\}$ twin in magnesium bounded by two types of boundaries, i.e. $\{10 \overline{1} 2\}$ interface and basal-prismatic facets. The migration of all boundary types is mediated by the motion of interfacial disconnections. It was shown that basal-prismatic interfaces play an important role in twin growth. The lengths of basal-prismatic facets remain constant during migration independently of the applied strain. In contrast, the $\{10 \overline{1} 2\}$ interfaces increase their lengths during growth.
\end{abstract}

DOI: 10.12693 /APhysPolA.128.661

PACS: $61.72 . \mathrm{Bb}, 61.72 . \mathrm{Mm}, 62.20 . \mathrm{F}-$

\section{Introduction}

Twinning is an important mode of deformation in materials with hexagonal close packed (hcp) structure. Significant twinning activity is caused by the lack of slip systems capable of accommodating an arbitrary deformation of the sample [1]. Different slip systems have significantly different critical resolved shear stresses in hcp materials [2] in contrast to cubic materials. Many slip systems are hard to activate providing room for deformation twinning.

A variety of twins have been observed in hcp materials [3]. The most frequent one is on the $\{10 \overline{1} 2\}$ planes accommodating tensile deformation along the $c$ direction in materials with $c / a<\sqrt{3}$. The twinning deformation is represented by shear, which takes place along the plane designated $K_{1}$ in the twinning direction $\eta_{1}$. Twinning mode is usually characterized by a set of four twinning elements $K_{1}, \eta_{1}, K_{2}, \eta_{2}$, where $K_{2}, \eta_{2}$ are the second undistorted but rotated plane and direction, respectively.

The twinning shear restores the initial crystal lattice but in a different crystallographic orientation. However, when the primitive cell contains more than one atom, the shear does not necessarily displace all atoms of the matrix into their correct positions in the twin. A combination of atomic shuffling (i.e. intracell atomic displacements that do not produce macroscopic deformation) and shear is then needed to restore the original crystal structure. The migration of twin boundaries is mediated by twinning disconnections [4-7]. The disconnection is an interfacial defect, which possesses the Burgers vector and produces a step on the interface. The motion of these disconnections,

\footnotetext{
* corresponding author; e-mail: ostapov@ipm.cz
}

and thus the twin boundary migration, is accomplished by a combination of shear and the accompanying atomic shuffling [8].

In the simplest case, the boundary between the twinned and un-twinned regions coincides with the $K_{1}$ plane. Nevertheless, there are many situations in which the interface is observed to deviate from the $K_{1}$ plane, for example at the tips of lenticular twins $[3,9$ 11]. Besides, basal-prismatic (BP) interfaces related to the $\{10 \overline{1} 2\}$ twin have been observed both experimentally $[12,13]$ and in atomistic simulations [14-19] in cobalt and magnesium.

In our previous paper [19], we reported an observation that BP interfaces do not increase their length during growth of octagonal shaped twin embryo. However, study [19] deals with only one type of applied strain. The goal of the present paper is the investigation of the evolution of a twin bounded by several types of interfaces under different applied strains.

\section{Model}

Magnesium was modelled by using the embedded atom method interatomic potential constructed by Liu et al. [20]. Calculations were performed in LAMMPS [21] and OVITO [22] was used for visualisation.

The simulation box was created by the following procedure. Initially a perfect crystal was created in a rectangular simulation block. The $x$ axis of the block was parallel to the [1011] direction, the $y$ axis was perpendicular to the (1012) plane, and the $z$ axis was parallel to the $[1 \overline{2} 10]$ direction. The block size was $608.784 \AA$ in $x$ direction, $579.853 \AA$ in $y$ direction and $19.236 \AA$ in $z$ direction. Periodic boundary conditions were applied in the $z$ direction. The atoms at the boundary of the simulated block in the $x$ and $y$ directions were free in the first 
relaxation and were held fixed in direction perpendicular to shear plane when further shear was applied.

Then a twinned embryo was created inside of simulation block by displacement of atoms to the twinned positions. The procedure is described with more details in [19]. The initial twin was bounded by four $\{10 \overline{1} 2\}$ planes and its size twin was $182.884 \AA$ in $x$ direction and $90.6833 \AA$ in $y$ direction. The minimization of the total potential energy of the block was performed using conjugate gradients in order to obtain the relaxed twin embryo. The embryo has three types of interfaces after the energy minimization: two twin interfaces on conjugate $\{10 \overline{1} 2\}$ planes and basal-prismatic facets as shown in Fig. 1a.

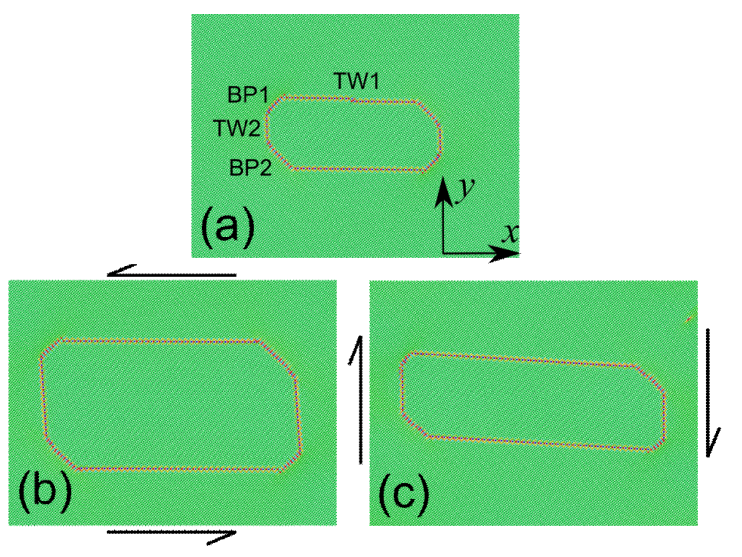

Fig. 1. (a) Initial configuration of twin embryo in the simulations; (b) and (c) Final configuration of twin embryo after shear strain applied to the simulation box in the directions shown by arrows.

In order to study the twin growth process, a shear was applied to the system. Two types of shear were examined. Each of them are favourable for growth one of two possible conjugate twin variants. The first one is a shear in the direction $x$ in the $y z$ plane and the other one is a shear in the direction $y$ in the $x z$ plane. The shear was applied by steps of $1 \%$ followed by energy minimization.

\section{Results and discussion}

The application of shear strain to the block leads to twin growth. The final state of the twin embryo after applied strain is shown in Fig. 1b,c. The octagonal shape of the initial twin remains preserved and the same types of interfaces still bound the spreading embryo. The migration of the twin interface is mediated by disconnections. Several types of disconnections were observed. Defects with two-layer steps $\left(b_{2 / 2}\right)$ glide along $\{10 \overline{1} 2\}$ planes. These disconnections are classical disconnections for $\{10 \overline{1} 2\}$ twinning in hexagonal close packed materials $[5,7,8]$. However, two types of defects are observed in BP interface; they have one-layer $\left(b_{1 / 1}^{\mathrm{BP}}\right)$ and two-layer steps $\left(b_{2 / 2}^{\mathrm{BP}}\right)$. Examples of these disconnections are shown in Fig. 2 .

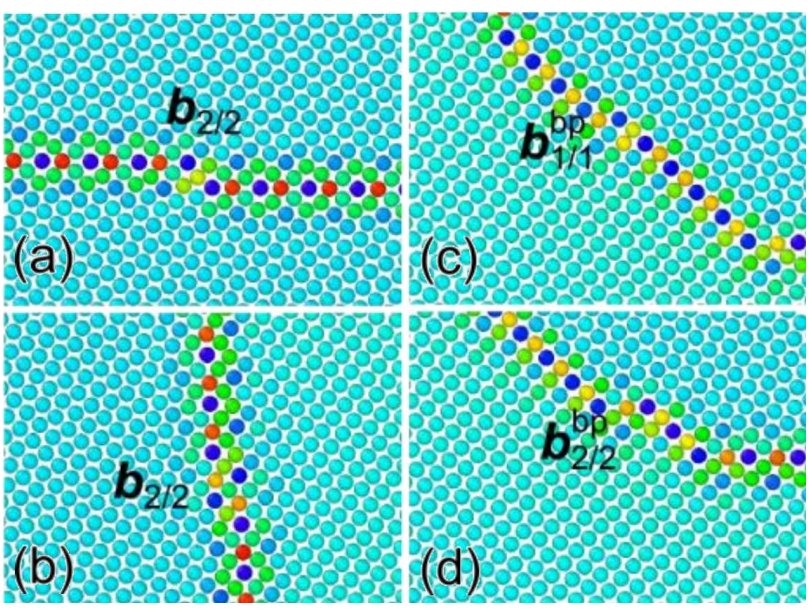

Fig. 2. Twinning disconnections in different types of twin-matrix interfaces (a) TW1 interface on (1012) plane; (b) Conjugate TW2 interface on (1012) plane; (c) and (d) basal-prismatic (BP1) and prismatic-basal (BP2) interfaces.

The twin misorientation across $\{10 \overline{1} 2\}$ plane is $86.3^{\circ}$. However, the misorientation across BP facet is $90^{\circ}$. The difference in misorientation is accommodated by disclination dipoles superimposed on the facets. Individual disclinations are located in the facet junctions.

The twin growth is accompanied by interactions between different types of interfacial defects. The disconnection reactions observed in our simulations are analogous to those recently reported by Barrett and El Kadiri [18, 23] and Ostapovets and Serra [19]. The reactions of disconnections with disclinations lead to changes in facet lengths. Thus, the interaction of a $b_{2 / 2}$ disconnection with a facet junction leads to the increase of $\mathrm{BP}$ facet length and decrease of $\{10 \overline{1} 2\}$ facet, whereas the interaction of disclination with $b_{2 / 2}^{\mathrm{BP}}$ produces an opposite effect, i.e. increase of $\{10 \overline{1} 2\}$ facet length and decrease of BP facet length. We never observed direct interaction of $b_{1 / 1}^{\mathrm{BP}}$ with facet junctions. Such disconnection interacts with each other producing $b_{2 / 2}^{\mathrm{BP}}$ (see Ref. [19] for details) before interaction with the junctions. New disconnection dipoles nucleated in the vicinity of facets junctions ensure the continuity of the growth process.

Figure 3 shows the evolution of facets lengths under applied shear strain. It can be seen that twin growth is accompanied by the elongation of TW1 and TW2 facets. The elongation values are dependent on the type of the applied strain. It is worth noting that migrations of TW1 and TW2 are connected to different twin modes. Shear strain in $x z$ plane is favorable for migration of TW 1 facet and shear in $y z$ plane is favorable for TW2 facet migration. The migration of TW1 facet leads mainly to the elongation of TW2 and vice versa. Initial asymmetry of embryo results in asymmetry of $\{10 \overline{1} 2\}$ facet elongation under different applied strains. The TW1 is initially shorter than TW2. Consequently, the twin deformation 


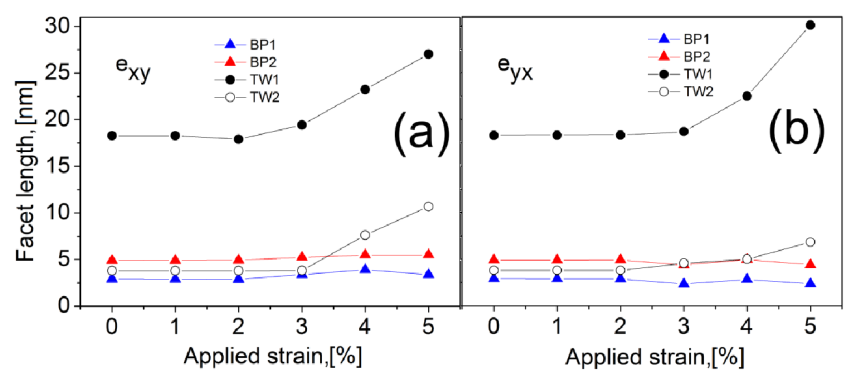

Fig. 3. Evolution of facet lengths under applied shear strain. (a) Shear in direction $x$ in $x z$ plane; (b) Shear in direction $y$ in $y z$ plane.

of the same volume needs more distant migration of TW2 in comparison to TW1.

The lengths of BP facets remain practically constant. The small variations of their lengths in Fig. 3 are not higher than changes produced by the interaction with one disconnection. These variations are continuously compensated by next disconnection reactions in the facet junctions. Several reasons exist for such behavior. The first one is the higher energy of BP interface $\left(173 \mathrm{~mJ} / \mathrm{m}^{2}\right)$ in comparison to $\{10 \overline{1} 2\}$ boundary $\left(122 \mathrm{~mJ} / \mathrm{m}^{2}\right)$. The second reason is the tendency to preserve coherency in the $\mathrm{BP}$ facet and avoid nucleation of misfit dislocations [18]. The coherency allows for BP interfaces to be mobile and actively participate in twin growth. The tendency to preserve the length of BP facets leads to decrease of the role of initially existed BP facets during twin growth. However, new BP facets can be nucleated, for instance, by interaction of twin boundary with twin dislocation [14]. Consequently, this tendency does not mean that BP facets disappear in large twins.

\section{Conclusions}

Growth of $\{10 \overline{1} 2\}$ twin embryo was investigated under two different applied strains. The twin embryo is bounded by different types of interfaces containing $\{10 \overline{1} 2\}$ facets as well as basal-prismatic facets. It was shown that the reactions between disconnections lead to balanced changes in lengths of individual facets in both considered cases. All types of interfaces migrate from their initial positions. The lengths $\{10 \overline{1} 2\}$ are changed during migration depending on the type of applied deformation. However, the basal-prismatic facets preserve their lengths during twin growth under different loading conditions.

\section{Acknowledgments}

A.O. acknowledges support from the Ministry of Education, Youth and Sports of the Czech Republic (grant number CZ.1.07/2.3.00/30.0063); Academy of Sciences of the Czech Republic, project no. RVO: 6808172; the Central European Institute of Technology (CEITEC) with research infrastructure supported by project CZ.1.05/1.1.00/02.006; A.S. acknowledges support from the Spanish Secretariat of Research, Development and Innovation under grant number FIS201239443-C02-02.

\section{References}

[1] M.H. Yoo, Metall. Mater. Trans. A 12, 409 (1981).

[2] W.B. Hutchinson, M.R. Barnett, Scr. Mater. 63, 737 (2010).

[3] J.W. Christian, S. Mahajan, Prog. Mater. Sci. 39, 1 (1995).

[4] R.C. Pond, in: Dislocations in Solids, Ed. F.R.N. Nabarro, North-Holland, Amsterdam 1989, p. 5.

[5] R.C. Pond, A. Serra, D.J. Bacon, Acta Mater. 47, 1441 (1999).

[6] R.C. Pond, X. Ma, Y.W. Chai, J.P. Hirth, in: Dislocations in Solids, Ed. J.P. Hirth, North-Holland, Amsterdam 2007, p. 225.

[7] A. Serra, R.C. Pond, D.J. Bacon, Acta Metall. Mater. 39, 1469 (1991).

[8] H.A. Khater, A. Serra, R.C. Pond, Philos. Mag. A 93, 1279 (2013).

[9] T. Braisaz, P. Ruterana, G. Nouet, Philos. Mag. A 76, 63 (1997).

[10] Y.J. Li, Y.J. Chen, J.C. Walmsley, R.H. Mathinsen, S. Dumoulin, H.J. Roven, Scr. Mater. 62, 443 (2010).

[11] J.X. Zhang, H.Q. Ye, J. Mater. Sci. Lett. 20, 9 (2001).

[12] X.Y. Zhang, B. Li, X. Wu, Y.T. Zhu, Q. Ma, Q. Liu, P.T. Wang, M.F. Horstemeyer, Scr. Mater. 67, 862 (2012).

[13] B.Y. Liu, J. Wang, B. Li, L. Lu, X.Y. Zhang, Z.W. Shan, J. Li, C.L. Jia, J. Sun, E. Ma, Nature Commun. 5, 3297 (2014).

[14] A. Serra, D.J. Bacon, Philos. Mag. A 73, 333 (1996).

[15] J. Wang, L. Liu, C.N. Tome, S.X. Mao, S.K. Gong, Mater. Res. Lett. 1, 81 (2013).

[16] B. Xu, L. Capolungo, D. Rodney, Scr. Mater. 68, 901 (2013).

[17] A. Ostapovets, R. Gröger, Model. Simul. Mater. Sci. Eng. 22, 025015 (2014).

[18] C.D. Barrett, H. El Kadiri, Acta Mater. 63, 1 (2014).

[19] A. Ostapovets, A. Serra, Philos. Mag. 94, 2827 (2014).

[20] X.Y. Liu, J.B. Adams, F. Ercolessi, J.A. Moriarty, Model. Simul. Mater. Sci. Eng. 4, 293 (1996).

[21] S. Plimpton, J. Comp. Phys. 117, 1 (1995).

[22] A. Stukowski, Model. Simul. Mater. Sci. Eng. 18, 015012 (2010).

[23] C.D. Barrett, H. El Kadiri, Acta Mater. 70, 137 (2014). 